\title{
Coordination, childhood weight gain and obesity
}

\section{Scott M. Montgomery PhD}

Previously published at www.cmaj.ca

$\infty \quad$ See related research article by Cairney and colleagues, page 1167

$\mathrm{O}$ besity can result in adverse neurologic sequelae such as diabetic retinopathy ${ }^{1}$ and cognitive decline and dementia, ${ }^{2}$ but there is evidence that some children at increased risk of obesity in adulthood already experience poorer neurologic function. ${ }^{3.4}$ In this issue of CMAJ, Cairney and colleagues report on their findings that children with possible developmental coordination disorder are at greater risk of unhealthy weight gain than those with better coordination. ${ }^{5}$ Several mechanisms should be considered to explain this increased risk, rather than assuming a simple cause-and-effect relationship. Also, some of these mechanisms will be relevant to a wider group of children than just those with developmental coordination disorder.

Previous research involving a British birth cohort showed that poorer physical control and coordination at ages 7 or 11 years was associated with an increased risk of obesity by 33 years of age. ${ }^{4}$ This finding is consistent with that of Cairney and colleagues, although the British study used several measures of coordination rather than focusing on the diagnostic criteria characterizing the developmental coordination disorder. The separate measures indicated poorer control and coordination at age seven years in $10 \%$ to $15 \%$ of the British cohort. ${ }^{4}$ Developmental coordination disorder is estimated to affect only $5 \%$ to $6 \%$ of children. ${ }^{6}$ Therefore, the association with unhealthy weight gain may not be limited to those with the more seriously impaired motor function characterized by the disorder.

Further evidence of this comes from three continuous scores relevant to motor function in the British cohort at age 11 years. ${ }^{4}$ The scores had an approximately linear association with risk of obesity in adulthood across all levels of motor function. Only one of the three scores appeared to show a disproportionately greater risk of obesity among children in the lowest functioning quartile; the test involved copying geometric designs accurately. This difference could be because the test was the most complex one and it did not test motor function alone. This assertion is supported by the fact that children with the lowest scores on this test also had the poorest cognitive function, an association not found with the other two tests.

The association of poorer coordination with unhealthy weight gain is not exclusively due to an increased risk among children with poorest motor function, such as those characterized as having developmental coordination disorder. The gradient of associations spans the population of ostensibly healthy children. However, children who have the more

\section{Key points}

- Children with coordination problems are more likely to have excess weight gain than children with better coordination.

- Although poor coordination itself may increase the risk of unhealthy weight gain, a variety of other mechanisms may be involved.

- If suboptimal neurologic function is a risk factor for unhealthy weight gain, then children with already limited function will be exposed to further risks of neurologic complications resulting from their excess weight.

severe impairments characterized by the disorder will be at a notably greater risk of unhealthy weight gain. It is therefore appropriate for Cairney and colleagues to emphasize the increased risk among children identified as having possible developmental coordination disorder.

The hypothesis that poorer coordination results in reduced physical activity and thus unhealthy weight gain is plausible. However, it may not be the only relevant mechanism, or the most important one. Compared with higher levels of motor function, even average levels are associated with a greater risk of obesity. Factors other than poor coordination may be responsible for increasing the risk of unhealthy weight gain. These factors may also be causes, or related to causes, of poorer coordination. For example, education has been recognized as an important risk factor for weight gain, with the biggest risk among children with the lowest level of education and poorest cognitive function. ${ }^{3}$ Some types of tasks that involve motor control are linked with cognitive function, as indicated by the test of copying designs discussed earlier. ${ }^{4}$ Do the children who have the cluster of more severe functional limitations characterized as developmental coordination disorder also have cognitive impairments, with educational and cultural correlates?

Low socio-economic status of the family is another recognized risk factor for unhealthy weight gain. ${ }^{3}$ It can be associated with adverse pregnancy outcomes, including low birth

Scott Montgomery is director of the Clinical Epidemiology and Biostatistics Unit, Örebro University Hospital, Örebro, Sweden, and is affiliated with the Department of Primary Care and Social Medicine, Imperial College, London, UK.

CMAJ 2010. DOI:10.1503/cmaj.100752

All editorial matter in CMAJ represents the opinions of the authors and not necessarily those of the Canadian Medical Association. 
weight, ${ }^{7}$ which in turn is a risk factor for neurosensory and coordination problems, including developmental coordination disorder, in offspring. ${ }^{8}$ The consequences of congenital neurodevelopmental impairment may be compounded by other factors related to economic disadvantage influencing unhealthy weight gain, because socio-economic adversity increases the risk of obesity among children ${ }^{9}$ and adults. ${ }^{10}$ Economic disadvantage and other family influences during childhood may also impair neurologic development, as signaled by delayed attainment of developmental milestones, ${ }^{11}$ and therefore result in impaired coordination or possibly worsen the effects of a congenital impairment. For these reasons, a high proportion of children found to have possible developmental coordination disorder through screening in later childhood may be from a relatively disadvantaged socio-economic background.

Other factors could influence both motor function and weight gain. Psychosocial stress has been identified as a risk factor for unhealthy weight gain in children, ${ }^{12,13}$ with potential adverse consequences for neurologic development and function. ${ }^{14}$ Family stress or its causes can also influence aspects of parenting relevant to physical activity that may set behavioural patterns that result in poor motor development and unhealthy weight gain. Children as young as three years old from stressed families have been observed to spend significantly more time watching television than children with less exposure to familial stress. ${ }^{13}$

The children with relatively poor coordination described by Cairney and colleagues clearly have an elevated risk for obesity later in life and might benefit from early interventions to tackle their excess weight gain and impaired motor function. However, perhaps we should extend our focus to a larger group of children with poorer coordination, not all of whom meet all of the diagnostic criteria for developmental coordination disorder. They, too, will be at increased risk of obesity.

Even if reduced participation in physical activity is the main cause of unhealthy weight gain in children with developmental coordination disorder, several nonmutually exclusive mechanisms are also likely to contribute to suboptimal development. It may not be enough to focus only on physical activity. A life-course approach is required to examine the long-term effects of multiple risk factors from early life onward that influence both physical function and weight gain.

The findings reported by Cairney and colleagues raise short- and longer-term public health concerns relevant to the obesity epidemic. If suboptimal neurologic function is a risk factor for unhealthy weight gain, then children with already limited function will be exposed to further risks of neurologic complications in later life such as diabetic retinopathy and dementia resulting from their excess weight.

Competing interests: Scott Montgomery is supported by a grant from the UK Economic and Social Research Council (grant no. RES-596-28-0001) to the International Centre for Life Course Studies in Society and Health for his work in investigating life-course influences on health.

\section{REFERENCES}

1. Said G. Diabetic neuropathy: a review. Nat Clin Pract Neurol 2007;3:331-40.

2. Whitmer RA, Gunderson EP, Barrett-Connor E, et al. Obesity in middle age and future risk of dementia: a 27 year longitudinal population based study. BMJ 2005;330:1360.

3. Chandola T, Deary IJ, Blane D, et al. Childhood IQ in relation to obesity and weight gain in adult life: the National Child Development (1958) Study. Int J Obes (Lond) 2006;30:1422-32.

4. Osika W, Montgomery SM. Physical control and coordination in childhood and adult obesity: Longitudinal Birth Cohort Study. BMJ 2008;337:a699.

5. Cairney J, Hay J, Veldhuizen S, et al. Trajectories of relative weight and waist circumference among children with and without developmental coordination disorder. CMAJ 2010;182:1167-72.

6. Gibbs J, Appleton J, Appleton R. Dyspraxia or developmental coordination disorder? Unravelling the enigma. Arch Dis Child 2007;92:534-9.

7. Muhajarine N, Vu LT. Neighbourhood contexts and low birthweight: social disconnection heightens single parents risks in Saskatoon. Can J Public Health 2009;100:130-4

8. Holsti L, Grunau RV, Whitfield MF. Developmental coordination disorder in extremely low birth weight children at nine years. J Dev Behav Pediatr 2002;23:9-15.

9. Wake M, Hardy P, Canterford L, et al. Overweight, obesity and girth of Australian preschoolers: prevalence and socio-economic correlates. Int J Obes (Lond) 2007; 31:1044-51.

10. Anand SS, Razak F, Davis AD, et al. Social disadvantage and cardiovascular disease: development of an index and analysis of age, sex, and ethnicity effects. Int $J$ Epidemiol 2006;35:1239-45.

11. Kelly Y, Sacker A, Schoon I, et al. Ethnic differences in achievement of developmental milestones by 9 months of age: The Millennium Cohort Study. Dev Med Child Neurol 2006;48:825-30.

12. Koch FS, Sepa A, Ludvigsson J. Psychological stress and obesity. J Pediatr 2008;153:839-44.

13. Stenhammar C, Olsson GM, Bahmanyar S, et al. Family stress and BMI in young children. Acta Paediatr 2010 Feb. 22. [Epub ahead of print]

14. Kikkert HK, Middelburg KJ, Hadders-Algra M. Maternal anxiety is related to infant neurological condition, paternal anxiety is not. Early Hum Dev 2010;86:171-7.

Correspondence to: Prof. Scott M. Montgomery, Clinical

Epidemiology and Biostatistics Unit, Örebro University Hospital,

70185 Örebro Sweden; scott.montgomery@ki.se

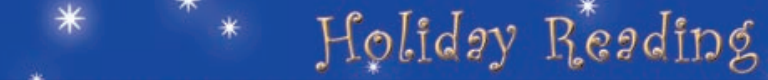 \\ …............................... \\ Call for papers}

"Hilarity and good humour ... help enormously in both the study and the practice of medicine." — William Osler

Twisted research, humorous reflections, witty rants, culturally current missives - we'll consider it all for CMAJ's 2010 Holiday Reading. Articles should be no longer than 1200 words; photographs and illustrations are welcome. Please submit your amusing missive online at http://mc.manuscriptcentral.com/cmaj, and specify in your cover letter that it's for the Holiday Reading section.

Deadline: October 1, 2010. 